\begin{tabular}{|l|l|}
\hline $\begin{array}{l}\text { Instituto de } \\
\text { Geriatria e Gerontologia }\end{array}$ & $\begin{array}{l}\text { Pan American Journal of Aging Research } \\
\text { PAJAR, Porto Alegre, v. 8, p. 1-9, jan.-dez. } 2020 \\
\text { ISSN-L: 2357-9641 }\end{array}$ \\
\hline http://dx.doi.org/10.15448/2357-9641.2020.1.37867 \\
\cline { 2 - 3 }
\end{tabular}

\title{
Risco para desenvolvimento de transtorno cognitivo em um grupo de idosos ativos
}

Risk for the development of cognitive disorder in a group of active elderly

Riesgo para desarrollo de trastorno cognitivo en un grupo de personas mayores activas

\author{
Maria Elisa Gonzalez \\ Manso ${ }^{1}$ \\ orcid.org/0000-0001-5446-233X \\ mansomeg@hotmail.com
}

Celia Maria Francisco ${ }^{1}$

orcid.org/0000-0002-0519-8058

celia.francisco@prof.saocamilo-sp.br

Renata Laszlo Torres ${ }^{1}$

orcid.org/0000-0001-9588-8070

relaszlo@gmail.com

\section{Mayara da Rocha \\ Camargo $^{1}$}

orcid.org/0000-0002-0641-842X

ma.cmg@hotmail.com

\section{Henrique Souza Barros \\ de Oliveira ${ }^{1}$ \\ orcid.org/0000-0002-9067-8581 \\ heeenry.barros@icloud.com}

\section{Diego Olympio Peixoto \\ Lopes $^{2}$}

orcid.org/0000-0001-8586-1640

diego_olympio@hotmail.com

Romário Daniel da Silva

Queiroz $^{2}$

orcid.org/0000-0001-8792-7770

d.romario5@gmail.com

Recebido em: 29/4/2020.

Aprovado em: 17/9/2020.

Publicado em: 13/11/2020.

\section{Resumo}

Objetivo: apresentar os fatores envolvidos no risco para o surgimento de transtornos cognitivos em um grupo de idosos ativos que participa de um Núcleo de Convivência para Idosos localizado na zona leste da cidade de São Paulo. Métodos: pesquisa desenvolvida a partir da aplicação de avaliação interdisciplinar a 68 idosos ativos entre os meses de janeiro a julho de 2019. Realizou-se a coleta de dados sociodemográficos, de funcionalidade e de morbidade, sendo utilizado o instrumento Miniexame do Estado Mental para categorização de dois grupos: idosos com risco e idosos sem risco para transtornos cognitivos. Esses grupos foram, então, comparados.

Resultados: observou-se que $38,2 \%$ dos idosos participantes apresentaram risco para transtornos cognitivos e que a variável idade avançada obteve associação significativa com esse risco: o avançar da idade aumenta o risco para o desfecho em 1,10 vezes. Observou-se ainda dado controverso quanto ao excesso de peso, o qual associou-se inversamente ao desfecho estudado.

Conclusão: para esse grupo de pessoas idosas, a idade avançada demonstrou ser fator de risco associado ao desenvolvimento de transtorno cognitivo, ao contrário do excesso de peso.

Palavras-chave: idoso, assistência integral à saúde, transtornos neurocognitivos, fatores de risco, testes de estado mental e demência, serviço social.

\section{Abstract}

Objective: present the factors involved in the risk for the emergence of cognitive disorders in a group of active elderly people who participate in an Elderly Living Center located on the east side of the city of São Paulo.

Methods: research developed from the application of an interdisciplinary assessment to 68 active elderly between the months of January to July 2019. Sociodemographic, functionality and morbidity data were collected, using the Mini-Mental State Examination instrument for the categorization of two groups: elderly with risk and elderly without risk of cognitive disorders. These groups were then compared.

Results: it was observed that $38.2 \%$ of the elderly participants were at risk for cognitive disorders and that the variable advanced age had a significant association with this risk: advancing age increases the risk for the outcome by 1.10 times. There was also controversial data regarding excess weight, which was inversely associated with the studied outcome.

Conclusion: for this group of elderly people, advanced age has been shown to be a risk factor associated with the development of cognitive disorder, as opposed to being overweight.

Keywords: aged, comprehensive health care, neurocognitive disorders, risk factors, mental status and dementia tests, social work.

\section{(c) (i)}

Artigo está licenciado sob forma de uma licença Creative Commons Atribuicão 4.0 Internacional. 


\section{Resumen}

Objetivo: presentar los factores involucrados en el riesgo de aparición de trastornos cognitivos en un grupo de personas mayores activas que participan en un Centro para Personas Mayores ubicado en el lado este de la ciudad de São Paulo.

Métodos: investigación desarrollada a partir de la aplicación de la evaluación interdisciplinaria a 68 ancianos activos entre los meses de enero a julio de 2019. Se recopilaron datos sociodemográficos, de funcionalidad y de morbilidad, utilizando el instrumento Mini-Mental State Examination para la categorización de dos grupos: ancianos en riesgo y ancianos sin riesgo de trastornos cognitivos. Estos grupos fueron, entonces, comparados. Resultados: se observó que el 38,2\% de los participantes de edad avanzada tenían riesgo de trastornos cognitivos y que la variable edad avanzada tenia una asociación significativa con este riesgo: conforme la edad avanza, aumenta el riesgo de aparecimiento del desenlace en 1,10 veces. Todavía, se encontró datos controvertidos sobre el exceso de peso, que se asoció inversamente con el desfecho estudiado.

Conclusión: para este grupo de personas mayores, la edad avanzada se comportó como un factor de riesgo asociado con el desarrollo del trastorno cognitivo, al contrario de tener sobrepeso.

Palavras clave: anciano, atención integral de salud, trastornos neurocognitivos, factores de riesgo, pruebas de estado mental y demencia, servicio social.

\section{Introdução}

O envelhecimento populacional é um fenômeno global. Os paises do mundo enfrentam um incremento tanto no número quanto na proporção de idosos: em 2019, a população mundial de pessoas idosas era de 703 milhões, podendo chegar, em 2050, a 1,5 bilhão. ${ }^{1}$ Já no Brasil, esse segmento etário foi estimado em 20 milhões em 2019, devendo alcançar 50 milhões até 2050. ${ }^{2}$ Esse fenômeno, denominado transição demográfica, é caracterizado pelo incremento proporcional no número de idosos devido à redução das taxas de natalidade e de mortalidade, sendo associado tanto aos avanços no desenvolvimento socioeconômico das populações quanto ao incremento de políticas públicas de saúde e de assistência social. Caracteriza-se, segundo a Organização Mundial de Saúde (OMS), indivíduos com idade igual ou superior a 60 anos, como idoso. Todavia, cabe ressaltar que esse limite mínimo pode variar conforme a formulação das políticas públicas e as condições de cada país. ${ }^{1.3}$

A transição demográfica vem sendo acompanhada por um importante desafio para a saúde pública, o predomínio de Doenças e Agravos Crônicos Não Transmissiveis (DANT) entre as pessoas idosas. As DANT encontram-se entre as principais causas de multimorbidades, de queda da qualidade de vida, mortalidade, declínio funcional e procura por serviços de saúde. ${ }^{4}$ Esse predomínio impõe um repensar na atenção à saúde da população idosa, com enfoque interprofissional, interdisciplinar e intersetorial, onde os cuidados de longo prazo e o suporte às familias se destacam. ${ }^{3}$

Dentre as DANT, a sindrome demencial é uma das causas frequentes de morbimortalidade entre os idosos. Denominada, a partir da edição do Manual Diagnóstico e Estatístico de Transtornos Mentais, $5^{a}$ edição (DSM-5) ${ }^{5}$ como transtorno neurocognitivo maior (major neurocognitive disorder), é caracterizada pelo comprometimento cognitivo importante em relação a um nível prévio de performance em um ou mais domínios, tais como: atenção, função executiva, aprendizado e memória, linguagem, percepção motora, cognição social. Esse declínio deve ser tão importante que comprometa a execução de tarefas de vida diária de forma independente, o que faz com que a pessoa necessite de auxilio, de forma progressiva. ${ }^{5}$ Por ser altamente influenciada por aspectos socioculturais no que tange à manutenção das capacidades dependentes da experiência, pode existir compensação inicial das perdas biológicas. ${ }^{67}$

Trata-se de uma sindrome clínica categorizada ou como degenerativa/primária do sistema nervoso central ou como não degenerativa/secundária a várias causas. Dentre as primárias, se enquadram a Doença de Alzheimer, Demência Frontotemporal, Demência dos Corpúsculos de Lewy como aquelas enfermidades que têm o transtorno neurocognitivo maior como manifestação principal, enquanto a Doença de Parkinson pode ter a demência como um sintoma. Já entre as secundárias surgem: a Demência Vascular, por Deficiência de Vitamina, as Tóxicas, as Metabólicas, as Infecciosas, as causadas por Trauma, dentre outras. ${ }^{7.8}$

Apesar dos avanços, não há terapia curativa, até o presente momento, para os transtornos cognitivos maiores primários. São afecções que 
causam impacto importante sobre a capacidade funcional, autonomia, participação social e profissional, saúde e qualidade de vida tanto dos idosos quanto de seus cuidadores e familiares, impondo desafios à equipe de saúde no que tange aos cuidados a serem oferecidos., ${ }^{8,9}$

Dentre esses transtornos, a Doença de Alzheimer é a mais frequente, afetando cerca de 25 milhões de pessoas em todo o mundo, tendo projeções para atingir mais de 100 milhões até 2050. Evidências apontam para maior prevalência dessa enfermidade em idosos que residem em países de média e baixa renda. ${ }^{9,10}$ Apesar disso, ainda há carência de estudos, principalmente, quando relacionados a fatores de risco que podem estar associados à frequência desses transtornos em pessoas idosas consideradas ativas e assintomáticas. A maioria das pesquisas ainda se relacionam a idosos diagnosticados com outras afecções correlacionadas ou asilados ${ }^{7,11-13}$. Entender esses aspectos pode contribuir sobremaneira para que a equipe interdisciplinar possa estabelecer planos de cuidados preventivos adequados a esse público.

Esta pesquisa tem como objetivo apresentar os fatores envolvidos no risco para o surgimento de transtornos cognitivos em um grupo de idosos ativos que participa de um Núcleo de Convivência para Idosos localizado na Zona Leste da cidade de São Paulo.

\section{Métodos}

Pesquisa transversal, exploratória, desenvolvida a partir da aplicação de avaliação multidimensional e interdisciplinar aos 68 idosos que participam ativamente de um Núcleo de Convivência de Idosos ( $\mathrm{NCl}$ ) localizado na Zona Leste da cidade de São Paulo.

Os $\mathrm{NCl}$ são serviços de proteção social básica, porta de entrada do Sistema Único de Assistência Social (SUAS). ${ }^{14}$ Para que um idoso frequente esses serviços, além da idade acima de 60 anos, deve encontrar-se em situação de vulnerabilidade e de risco pessoal e/ou social caracterizada por vivências de isolamento social e de ausência de acesso a serviços. Esses $\mathrm{NCl}$ são locais onde se promovem atividades socioeducativas-culturais que buscam facilitar a construção e a reconstrução das histórias e das vivências individuais e coletivas, a fim de promover o convívio com a familia e com a comunidade. Tais atividades buscam um envelhecimento mais ativo e saudável, motivando esses idosos a terem novos projetos de vida, além de promover saúde e prevenir doenças, isolamento e asilamento. ${ }^{15,16}$

Todos os idosos que compõem esse grupo pesquisado participavam ativamente das práticas realizadas no espaço do $\mathrm{NCl}$ entre os meses de janeiro a julho de 2019, daí sua inclusão no estudo. Foram excluidos os idosos acamados, os quais o $\mathrm{NCl}$ assiste em visitas domiciliares, bem como um idoso que, apesar de participar ativamente, já tinha diagnóstico de transtorno cognitivo maior. Essas pessoas tomaram ciência do ingresso opcional, do sigilo dos seus dados e que sua não aceitação não interferiria no serviço prestado pelo $\mathrm{NCl}$, tendo assinado um Termo de Consentimento Livre e Esclarecido.

Realizou-se, então, entrevista individual para coleta de dados sociodemográficos, de funcionalidade e de morbidade. A variável dependente foi o risco para presença de déficit cognitivo, mensurada pelo Mini Exame do Estado Mental (MEEM). ${ }^{17}$ Para esse instrumento empregou-se pontos de corte recomendados pelo Ministério da Saúde (MS) brasileiro, segundo o grau de escolaridade. ${ }^{18}$ Neste estudo optou-se por adotar a expressão "com risco para transtorno cognitivo" para idosos que apresentaram escores alterados e "sem risco para transtorno cognitivo" para os que não apresentaram, com base nos pontos de corte propostos pelo ministério.

O MEEM foi desenvolvido por pesquisadores americanos em 1975, em virtude da carência de uma ferramenta capaz de avaliar o risco para déficits cognitivos de forma padronizada, simplificada, reduzida e de rápida aplicação na prática das equipes de saúde. No entanto, esse instrumento somente alcançou popularidade na década de 1980, após a publicação do estudo epidemiológico que permitiu o desenvolvimento de pontos de corte com base na idade e no grau de escolarida- 
de. Assim, tornou-se o instrumento mais utilizado e empregado tanto na prática dos profissionais que atuam na atenção à saúde da pessoa idosa quanto em estudos para rastreio de transtornos cognitivos em indivíduos adultos e idosos. O MEEM é composto por seções capazes de mensurar funções cognitivas, tais como: orientação; memória imediata; atenção; cálculo; capacidade de nomeação; de obediência a um comando verbal e a um escrito, de redação livre de uma sentença e de cópia de um desenho complexo. Não é um teste de diagnóstico e sim de rastreio, indicando funções que necessitam ser melhor investigadas, sendo um dos poucos validados e adaptados para a população brasileira. Em relação ao escore, esse pode variar entre zero e 30 pontos baseados em itens dicotômicos. Ressalta-se ainda que os pontos de corte podem variar dependendo do autor e/ ou recomendação de cada país. 19,20,21

As variáveis independentes foram divididas em sociodemográficas (sexo; idade; estado conjugal; grau de escolaridade e com quem reside); funcionalidade (índice de dependência para as Atividades Básicas e Instrumentais de Vida Diária - Katz Índex/Lawton Índex respectivamente) ${ }^{22}$ e morbidades categorizadas, de acordo com a Classificação Internacional de Doenças (CID 10). ${ }^{23}$

A mensuração da capacidade funcional para ABVD representa a aptidão para realizar tarefas necessárias para o cuidar de si, como comer, tomar banho, vestir-se, ir ao banheiro, andar, dentro outras. Já as AIVD referem-se a atividades mais complexas, influenciadas pela cultura, tais como preparar a comida, lavar as roupas, cuidar da casa, fazer compras, como exemplos. ${ }^{22}$

Quanto às medidas antropométricas, utilizou- se a classificação do estado nutricional que leva em consideração as modificações na composição corporal próprias do envelhecimento, por meio de um estadiômetro portátil com trena retrátil para mensuração da altura e uma balança eletrônica portátil para mensuração do peso.

Inicialmente, os dados foram tabulados e categorizados no programa Excel e posteriormente analisados pelo Software Statistical Package for Social Sciences (SPPS). A estatística descritiva foi realizada determinando-se indicadores de frequência relativa e absoluta das variáveis categóricas e medidas de tendência central e dispersão para as variáveis numéricas. A associação entre os grupos com risco para transtorno cognitivo e as variáveis independentes foi calculada utilizando o teste de Qui-Quadrado e o teste t-Student. As variáveis que apresentaram significância foram então selecionadas para compor o modelo de regressão logística através do método de Stepwise Forward, com intuito de identificar os fatores que influenciam o desenvolvimento de transtorno cognitivo. Considerou-se significância estatística para todos os testes quando $\mathrm{p}<0,05$.

Este estudo foi aprovado pelo Comitê de Ética e Pesquisa da Pontifícia Universidade Católica de São Paulo (PUC-SP), sob parecer número 2.626.054, CAAE: 87307118500005482.

\section{Resultados}

O grupo foi composto, em sua maioria, por idosos do sexo feminino ( $n=61,89,7 \%$ ), cuja média e mediana de idade foram iguais a 74,8 e 75 anos, respectivamente, variando entre 61 e 88 anos de idade. Em relação à situação conjugal, a maior parte eram viúvos $(n=30,44,1 \%)$, seguidos por idosos casados ( $n=23,33,8 \%$ ). Quanto à moradia, $64,7 \%$ ( $n=44$ ) dos idosos do grupo residiam com algum familiar ou amigo, sendo cônjuges e/ou filhos ( $n=42,61,7 \%$ ) os mais prevalentes. O nível de escolaridade predominante no grupo foi o ensino fundamental incompleto $(n=39,57,4 \%)$. Esses dados podem ser mais bem visualizados na Tabela 1. 
TABELA 1 - Principais dados sociodemográficos e de funcionalidade, grupo de idosos assistidos pelo $\mathrm{NCl}$, cidade de São Paulo, 2019

\begin{tabular}{lll}
\multicolumn{1}{c}{ Variáveis } & $\mathbf{n}$ & $\%$ \\
\hline Sexo & 61 & 89,7 \\
$\quad$ Feminino & 7 & 10,3 \\
$\quad$ Masculino & & \\
Situação conjugal & 30 & 44,1 \\
$\quad$ Viúvos & 23 & 33,8 \\
Casados & 15 & 22,1 \\
Outros & & \\
Nivel de Escolaridade & 5 & 7,4 \\
$\quad$ Analfabetos & 39 & 57,4 \\
Ensino fundamental incompleto & 13 & 19,1 \\
Ensino fundamental completo & 11 & 16,1 \\
Outros & & \\
ABVD* & & \\
$\quad$ Independente para ABVD & 43 & 63,2 \\
Dependência leve para ABVD & 25 & 36,8 \\
AIVD** & & \\
Independente para AIVD & 67 & 98,5 \\
Dependência leve para AIVD & 1 & 1,5 \\
\hline
\end{tabular}

"ABVD: Atividades Básicas de Vida Diária; "*AIVD: Atividades Instrumentais de Vida Diária.

Sobre a funcionalidade, notou-se que a predominância foi de idosos com capacidade funcional preservada, tanto para Atividades Básicas de Vida Diária (ABVD), quanto para Atividades Instrumentais de Vida Diária (AIVD), conforme também pode ser visto na Tabela 1. Porém, 25 (36,8\%) desses idosos apresentavam dependência para a realização de ABVD, principalmente relacionadas à mobilidade restrita pela presença de dor.

Quanto à capacidade cognitiva, observou-se que $38,2 \%(n=26)$ dos participantes apresentaram risco para transtorno cognitivo, atingindo ponto de corte abaixo dos padrões do MS em relação ao nivel de escolaridade. Ficaram acima desses padrões, sendo, portanto, classificados como sem risco para transtorno cognitivo, 42 idosos (61,8\%).

A Tabela 2 apresenta as principais DANT encontradas para esse grupo, sendo que a hipertensão arterial sistêmica, a dislipidemia, a dor crônica, as tireoidopatias e a osteoporose foram as mais prevalentes. A média de DANT encontrada por idoso foi de 3.5, com mediana de 3 e variando entre nenhuma a 8 /idoso.
TABELA 2 - Principais DANT categorizadas segundo CID-10, grupo de idosos assistidos pelo $\mathrm{NCl}$, cidade de São Paulo, 2019

\begin{tabular}{|c|c|c|}
\hline Sistema & DANT & $\begin{array}{l}\mathrm{N}^{\circ} \mathrm{de} \\
\text { idosos }\end{array}$ \\
\hline \multirow{3}{*}{ Cardiovascular } & $\mathrm{HAS}^{*}$ & 47 \\
\hline & Dislipidemia & 36 \\
\hline & Cardiomiopatia & 10 \\
\hline \multirow{4}{*}{$\begin{array}{l}\text { Metabólico e } \\
\text { hormonal }\end{array}$} & Tireoidopatias & 22 \\
\hline & Diabetes Mellitus & 15 \\
\hline & Sobrepeso & 14 \\
\hline & Obesidade & 3 \\
\hline Neurológico & $A V E^{* *}$ & 5 \\
\hline $\begin{array}{l}\text { Musculoesquelético } \\
\text { e articular }\end{array}$ & Osteoporose & 16 \\
\hline \multirow{4}{*}{ Outros } & Neoplasias & 10 \\
\hline & $\mathrm{DRC}^{+}$ & 3 \\
\hline & Dor crônica & 34 \\
\hline & Outras & 11 \\
\hline
\end{tabular}

*HAS: Hipertensão Arterial Sistêmica; * AVE: Acidente Vascular-encefálico; ‘DRC: Doença Renal Crônica

Em relação à análise comparativa das variáveis sociodemográficas e de funcionalidade pesquisadas nos grupos com e sem risco para transtorno cognitivo mensurado pelo MEEM, observou-se que apenas o parâmetro idade avançada obteve diferença estaticamente significativa. Os idosos com pontuação no MEEM sem risco para transtorno cognitivo atingiram média de idade igual a 73.5 anos, enquanto aqueles com potencial risco tinham média de idade igual a 76,8 anos $(p=0,047)$. Aplicada regressão logística, notou-se que a idade avançada acrescenta 1,10 vezes mais chances (OR: 1,10; IC95\%: 1,01-1,20; $\mathrm{p}=0,024$ ) de desenvolvimento de transtorno cognitivo nestes idosos.

Quanto às DANT, somente foi encontrada associação significativa entre excesso de peso e idosos sem risco para transtorno cognitivo $(p=0,039)$.

\section{Discussão}

Quando analisada a distribuição das pessoas idosas que frequentam o $\mathrm{NCl}$ pesquisado, obser- 
va-se um padrão quanto ao sexo que não difere do encontrado para a população idosa brasileira. Várias pesquisas demonstram que as mulheres tendem a ser mais preocupadas com sua saúde e avessas ao risco, o que faz com que procurem e participem mais de serviços de promoção à saúde, como os NCl. Padrão semelhante às características da população brasileira também é visto neste grupo no que diz respeito ao nivel de escolaridade. ${ }^{2,24}$

Já a situação conjugal do grupo reflete os achados da literatura. Em relação à moradia, o arranjo mais representativo encontrado na população idosa brasileira é o casal que reside com filhos e outros parentes, como aqui encontrado. A literatura ressalta que esse tipo de arranjo pode não ser uma opção das pessoas e sim resultado de situações sócio-histórico-culturais e econômicas que podem interferir na qualidade de vida. Desde a década de 1990 observa-se que os idosos, principalmente as mulheres que têm sua renda advinda de aposentadorias e pensões, podem oferecer suporte financeiro à família, o que pode explicar a coabitação. O tipo de arranjo familiar também sofre influência da escolaridade, sendo que idosos com menor escolaridade tendem a morar com seus parentes. ${ }^{2.25}$

Evidências apontam para a associação entre fatores sociodemográficos e do estilo de vida, tais como: (i) ser do sexo feminino, (ii) ter baixa escolaridade, (iii) ser sedentário, (iii) apresentar condição socioeconômica desfavorável e (iv) existir situação de fragilidade no idoso, como fatores de risco para o desenvolvimento de transtornos

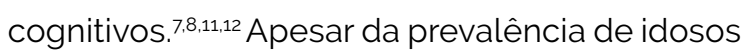
do sexo feminino, com baixo grau de escolaridade e vulnerabilidade social nesse grupo de idosos, não foram encontradas associações significativas entre essas variáveis e a pontuação do MEEM.

Uma pesquisa ${ }^{23}$ demonstra que o maior grau de dependência para essas habilidades é encontrado acima dos 70 anos de idade, o que não foi encontrado nesse grupo pesquisado onde a grande maioria é totalmente independente nas AIVD e ABVD.

Porém, notou-se que ter dor crônica limita a independência dessas pessoas idosas, interfe- rindo no desempenho das ABVD e fazendo com esses idosos, apesar de ativos, já necessitem de algum auxilio, principalmente do uso de órteses como bengalas.

Sobre as DANT apresentadas por esses idosos, salienta-se a associação encontrada entre elas. Assim, a presença de hipertensão arterial combinada com a dislipidemia relaciona-se a maior probabilidade de ocorrência de eventos cardiovasculares, tais como infarto do miocárdio e acidente vascular-encefálico. Ademais, a presença de multimorbidade vincula-se frequentemente à presença de polifarmácia, à maior probabilidade de surgimento de efeitos colaterais da medicação e de interações medicamentosas, bem como maior probabilidade de abandono do tratamento, acarretando diminuição da qualidade de vida da pessoa idosa, potencializando os riscos de desfechos graves. ${ }^{26}$

Outro fator é a presença de DANT, principalmente a hipertensão arterial, e sua associação com transtornos cognitivos. Em 2019, foi publicada pesquisa ${ }^{24}$ que observou relação entre o aumento dos níveis pressóricos e o declínio cognitivo progressivo, sendo que o controle rigoroso dos níveis pressóricos foi estatisticamente significativo para redução do risco para transtornos cognitivos maiores. Contudo, não foi possivel determinar se essa medida é benéfica para outros tipos de demências além da vascular. Neste grupo pesquisado, nem a hipertensão arterial nem outras DANT, exceto o sobrepeso, relacionaram-se a pontuação obtida pelo MEEM.

Interessante ressaltar a associação obtida entre excesso de peso e ausência de risco para transtorno cognitivo para esse grupo pesquisado. Há controvérsias importantes na literatura no que tange à associação entre o excesso de peso ou à presença de obesidade com os transtornos cognitivos maiores, as quais ainda não foram sanadas. O estudo Whitehall II, ${ }^{27}$ considerado uma das maiores pesquisas de coorte populacional, investigou a associação entre alterações do índice de massa corporal (IMC) e a ocorrência de demência, encontrando associação entre obesidade (caracterizada por $\mathrm{IMC}>30 \mathrm{~kg} / \mathrm{m}^{2}$ ) presente aos 50 anos de idade e desenvolvimento posterior 
de transtorno cognitivo, porém, tal associação não foi reproduzida para outras faixas etárias. Há artigos, entretanto, como o realizado com dados retrospectivos de 2 milhões de pacientes com mais de 40 anos durante duas décadas, ${ }^{28}$ que mostram que o aumento do IMC pode ser considerado um fator protetor para o surgimento de transtorno cognitivo, o que parece ocorrer nesse grupo de idosos aqui descrito.

O MEEM permanece sendo o instrumento de rastreio do estado cognitivo mais utilizado em todo o mundo, isoladamente ou em conjunto com outros instrumentos mais amplos. Quando da sua aplicação a esse grupo de idosos ativos, encontrou-se 26 (38,2\%) pessoas idosas com risco para transtorno cognitivo, porcentagem considerada elevada em relação a outros grupos populacionais. ${ }^{11,12,29}$

Considera-se que um dos principais fatores associados ao potencial risco de desencadeamento de transtorno cognitivos em idosos seja a idade avançada, especialmente quando associada à multimorbidade, ao uso de polifarmácia e à vulnerabilidade social, ,8,11,28 achado corroborado por esta pesquisa. Nesse grupo de idosos portadores de comorbidades em associação e importante vulnerabilidade social, fator de sua inclusão no $\mathrm{NCl}$, observou-se associação entre o avançar da idade e a pontuação do MEEM, sendo que a cada um ano de idade houve aumento de 1,10 vezes o risco para transtorno cognitivo.

Frisa-se, porém, que alguns pesquisadores têm apontado que a utilização do MEEM em pessoas saudáveis não teria efeito preditor do declínio cognitivo, detectando apenas déficits já presentes e ainda não detectados,30,33 fato preocupante tendo em vista a vulnerabilidade social das pessoas assistidas e o desconhecimento, tanto pela equipe técnica do $\mathrm{NCl}$ quanto pelos próprios idosos e seus familiares, dos declínios cognitivos levantados por esta pesquisa.

Deve-se ressaltar que esta pesquisa apresenta limitações tanto pelo desenho do estudo quanto pelo grupo pesquisado, o que não permite generalizações. Outra limitação se refere às próprias particularidades do instrumento MEEM, pois há controvérsias sobre o uso da escolaridade para estabelecer pontos de corte, bem como diferentes padronizações desses.

\section{Conclusão}

Esta pesquisa evidenciou os principais fatores envolvidos no surgimento de transtornos cognitivos em um grupo de idosos participantes de um $\mathrm{NCl}$ localizado na cidade de São Paulo, explicitando como principal fator de risco a presença de idade avançada, sendo que para o idoso cada ano a mais de vida leva ao incremento de 1,1 vezes na probabilidade de apresentar déficit cognitivo. Já o excesso de peso apareceu, para este grupo, associado à ausência de risco para transtorno cognitivo avaliado pelo MEEM, fato controverso na literatura e que carece de mais estudos.

Contudo, salienta-se ainda que, grupos de idosos com participação ativa em núcleos de convivência social, como aqui descrito, estão em processo contínuo de estímulo das habilidades sociais e cognitivas, podendo gerar influência sobre os resultados aqui apresentados.

Para a equipe de saúde, a relevância deste estudo está relacionada à ampliação da discussão sobre fatores relacionados aos transtornos cognitivos, que podem embasar um plano de cuidados voltado às necessidades dessas pessoas e de seus familiares, tanto preventivos, quanto de diagnóstico e de reabilitação.

\section{Referências}

1. United Nations. World Population Ageing 2019. [lnternet]. Department of Economic and Social Affairs, Population Division; 2020 [cited 2019 Dec 02]. Available at: https://www.un.org/en/development/desa/ population/publications/pdf/ageing/WorldPopulationAgeing2019-Report.pdf.

2. Instituto Brasileiro de Geografia e Estatística. Projeções da população [Internet]. Rio de Janeiro: Instituto Brasileiro de Geografia e Estatística; 2018 [cited 2019 Dec 02]. Available at: https://www.ibge.gov.br/estatisticas/sociais/populacao/9109-projecao-da-populacao. html? =\&t=resultados.

3. World Health Organization. Integrated Care for Older People (ICOPE): Guidance on person-centered assessment and pathways in primary care. Geneva: World Health Organization; 2019 [cited 2019 Dec 02]. Available at: https:// apps.who.int/iris/bitstream/handle/10665/326843/ WHO-FWC-ALC-19.1-eng.pdf;jsessionid=F9437A68FD62175ECAF733B B287715B2? sequence $=17$. 
4. Manso MEG, Sousa JRP, Oliveira HSB. Análise do perfil neuro geriátrico de um grupo de idosos pertencentes a um plano de saúde do município de São Paulo, Brasil. Rev Kairós-Gerontol. 2018;21(2):215-226.

5. American Psychiatric Association. Manual Diagnóstico e Estatístico de Transtornos Mentais: DSM-5/ 5. ed. Porto Alegre: Artmed; 2014.

6. Bernardo LD, Carvalho CRA. The role of cultural engagement for older adults: an integrative review of scientific literature. Rev. bras. geriatr. Gerontol. 2020;23(6): e190141. Epub. https://doi.org/10.1590/1981$\underline{22562020023.190141}$

7. Fernandes JSG, Andrade MS. Revisão sobre a Doença de Alzheimer: diagnóstico, evolução e cuidados. Psic., Saúde \& Doenças. 2017:18(1):131-140. Epub. http://dx.doi. org/10.15309/17psd180111

8. Andrea MCL, Rosa MMC. Demencia. Rev Act Clín. 2013:35:1810-14.

9. Oliveira IAG, Caetano R, Steffen RE, Biz NA. A systematic review of economic evaluations of the use of memantine alone or combined with donepezil for moderate to severe Alzheimer's disease. Rev Bras Geriatric Gerontol. 2019;22(4):e190002. https://doi. org/10.1590/1981-22562019022.190002.

10. Pelegrini LNC et al. Diagnóstico de demência e disfunção cognitiva em idosos na atenção primária à saúde: uma revisão sistemática. Dement. neuropsychol. [online]. 2019;13(2):144-153.

11. Ribeiro RCHM, Werneck AL, Cesarino CB et al. Estado Mental dos Idosos em Hemodiálise no Serviço de Nefrologia. Rev enferm UFPE on line. 2019;13(5):119220. https://doi.org/10.5205/1981-8963-v13i05a23876 6p1192-1201-2019

12. Wannmacher L. Demência: evidências contemporâneas sobre a eficácia dos tratamentos. Ciênc. saúde coletiva. 2020;25(2):603-611. Epub. https://doi. org/10.1590/1413-81232020252.02042018

13. Zimmermmann IMM, Leal MCC, Zimmermann RD, Marques APO. Idosos institucionalizados: comprometimento cognitivo e fatores associados. Geriatr Gerontol Aging. 2015:9(3):86-92. https://doi.org/10.5327/Z24472115201500030003 .

14. Lei No 8.742, de 7 de dezembro de 1993. Dispõe sobre a organização da Assistência Social e dá outras providências. [Internet]. 1993 [cited 2020 Jan 10]. Available at: http://Www.planalto.gov.br/ccivil_03/LEIS/L8742.htm.

15. Ministério do Desenvolvimento Social e Combate à Fome (MDS). Tipificação Nacional de Serviços Socioassistenciais. MDS: Brasília; 2014.

16. Derhun FM, Scolari GAS, Castro VC, Salci MA, Baldissera VDA, Carreira L. O centro de convivência para idosos e sua importância no suporte à família e à rede de atenção à saúde. Esc Anna Nnery. 2019;23(2):e $20180156 .$. https://doi.org/10.1590/2177-9465-ean-2018-0156

17. Rosen WG, Mohs RC, Davis KL. A new rating scale for Alzheimer's disease. American Journal of Psychiatry. 1984:141(11):1356-1364. https://doi.org/10.1176/ ajp.141.11.1356.
18. Ministério da Saúde, MS. Protocolo clínico e diretrizes terapêuticas: Doença de Alzheimer. Brasilia - DF: Ministério da Saúde; 2010 [cited 2029 dec 10]. Available at: http://bvsms.saude.gov.br/bvs/saudelegis/sas/2010/ prt0491_23_09_2010.html.

19. Brucki SMD, Nitrini R, Caramelli P, Bertolucci PHF, Okamoto IG. Sugestões para o uso do mini-exame do estado mental no Brasil. Arq Neuropsiquiatr. 2003:61(3-b):777-781. https://doi.org/10.1590/S0004282X2003000500014.

20. Gomes LMC, Martins LNSL. Avaliação Cognitiva e do Humor. In Manso MEG, Biffi ECA, organizadores. Geriatria: Manual da Liga de Estudos do Processo de Envelhecimento. São Paulo, SP: Martinari; 2015.

21. Melo DM, Barbosa AJG. O uso do Miniexame do Estado Mental em pesquisas com idosos no Brasil: Uma revisão sistemática. Ciênc. Saúde Coletiva. 2015;20(12):3865-3876. https://doi.org/10.1590/1413812320152012.06032015.

22. Dias AL, Rodrigues TC. Avaliação da Capacidade Funcional Avaliação Cognitiva e do Humor. In Manso MEG, Biffi ECA, organizadores. Geriatria: Manual da Liga de Estudos do Processo de Envelhecimento. São Paulo, SP: Martinari; 2015.

23. World Organization Health. International Statistical Classification of Diseases and Related Health Problems - ICD [Internet]. Geneva: World Organization Health; 2013 [cited 2019 Dec 02]. Available at: https://www.cid10.com.br/.

24. Mira BC, Ferreira AMR, Ozela CS et al. Determinantes Socioeconômicos e Comportamentais que permeiam o Envelhecimento Ativo dos Idosos de um Centro Comunitário de Convivência. Rev Fund Care Online. 2019;11(5):1122-1128. https://doi.org/10.9789/21755361.2019.v11i5.1122-1128.

25. Pereira AG, Alves LC. Condição de vida e saúde dos idosos: uma revisão bibliográfica. Campinas, SP: Núcleo de Estudos de População "Elza Berquó"; 2016.

26. Manso MEG, Osti AV, Borrozino NF, Maresti LTP. Avaliação Multidimensional do Idoso: resultados em um grupo de individuos vinculados a uma operadora de planos de saúde. Kairós - Gerontologia. 2018;21(1):191-211.

27. Vicario A, Coca A, Gasecki D, Zaninelli A, Lovic D, Manios E, et al. Effects of antihypertensive treatment on cognitive decline. Update on hypertension management. European Society of Hypertension. Sci Newsl [Internet]. 2019 [cited 2019 Dec 02];20(73):1-2. Available at: https://www.eshonline.org/esh-content/ uploads/2020/01/Newsletter-ESH-73_antihypertensive-treatment_rev3_OK.pdf.

28. Ferrie, J. E., Head, J., Shipley, M. J., Vahtera, J., Marmot, M. G. and Kivimäki, M. BMI, Obesity, and Sickness Absence in the Whitehall II Study. Obesity. 2017:15: 1554-1564. doi:10.1038/oby.2007.184

29. Qizilbash N, Gregson J, Johnson ME, Pearce N, Douglas I, Wing $\mathrm{K}$ et al. BMI and risk of dementia in two million people over two decades: a retrospective cohort study. The Lancet. 2015;3(6):431-436. https:// doi.org/10.1016/S2213-8587(15)00033-9. 
30. Castro-Costa E, Dewey ME, Uchôa E, Firmo JOA, Lima-Costa MF, Stewart R. Trajectories of cognitive decline over 10 years in a Brazilian elderly population: the Bambu cohort study of aging. Cad. Saúde Pública. 2011;27(3) s345-S350. https://doi.org/10.1590/S0102$311 \times 2011001500004$.

31. Santos CS, Bessa TA, Xavier AJ. Fatores associados à demência em idosos. Ciên. Saúde Coletiva. 2020;25(2):603-611. https://doi.org/10.1590/141381232020252.02042018.

32. Mota E, Banhato EFC, Silva KCA, Cupertino APFB. Triagem cognitiva: comparações entre o mini mental e o teste de trilhas. Est. Psicol. 2008;25(3):353-359. https://doi.org/10.1590/S0103-166X2008000300004.

33. Machado JC, Ribeiro RCL, Mitre RMC, Leal PFG. Declínio cognitivo de idosos e sua associação com fatores epidemiológicos em Viçosa, Minas Gerais. Rev. Bras. Geriatr. Gerontol. 2011:14(1):109-121. https://doi. org/10.1590/S1809-98232011000100012.

\section{Endereço para correspondência}

Maria Elisa Gonzalez Manso

Av. Nazaré, 1501

Ipiranga, 04262200

São Paulo, SP, Brasil 